\title{
LEFSCHETZ THEOREMS FOR TAMELY RAMIFIED COVERINGS
}

\author{
HÉLÈNE ESNAULT AND LARS KINDLER \\ (Communicated by Romyar T. Sharifi)
}

\begin{abstract}
As is well known, the Lefschetz theorems for the étale fundamental group of quasi-projective varieties do not hold. We fill a small gap in the literature showing they do for the tame fundamental group. Let $X$ be a regular projective variety over a field $k$, and let $D \hookrightarrow X$ be a strict normal crossings divisor. Then, if $Y$ is an ample regular hyperplane intersecting $D$ transversally, the restriction functor from tame étale coverings of $X \backslash D$ to those of $Y \backslash D \cap Y$ is an equivalence if dimension $X \geq 3$, and is fully faithful if dimension $X=2$. The method is dictated by work of Grothendieck and Murre (1971). They showed that one can lift tame coverings from $Y \backslash D \cap Y$ to the complement of $D \cap Y$ in the formal completion of $X$ along $Y$. One has then to further lift to $X \backslash D$.
\end{abstract}

\section{INTRODUCTION}

Let $X$ be a locally noetherian scheme, let $Y$ be a closed subscheme, and let $X_{Y}$ be the formal completion of $X$ along $Y$. Recall (see [7, X.2, p. 89]) that the condition Lef $(X, Y)$ holds if for every open neighborhood $U$ of $Y$ and every coherent locally free sheaf $E$ on $U$, the canonical map $H^{0}(U, E) \rightarrow H^{0}\left(X_{Y}, E_{X_{Y}}\right)$ is an isomorphism. For the condition $\operatorname{Leff}(X, Y)$, one requires in addition that every coherent locally free sheaf on $X_{Y}$ is the restriction of a coherent locally free sheaf on some open neighborhood $U$ of $Y$.

Assume $X$ is defined over a field $k$ and is proper. Let $D$ be another divisor, which has no common component with $Y$, such that $D \hookrightarrow X$ and $D \cap Y \hookrightarrow Y$ are strict normal crossings divisors (Definition 2.1). Let $\bar{y} \rightarrow Y \backslash D \cap Y$ be a geometric point. We define the functoriality morphism

$$
\pi_{1}^{\text {tame }}(Y \backslash D, \bar{y}) \rightarrow \pi_{1}^{\text {tame }}(X \backslash D, \bar{y})
$$

between the tame fundamental groups [13, $\S 7$ ]. If $\operatorname{char}(k)=0$, this is the usual functoriality morphism between the étale fundamental groups of $Y \backslash D$ and $X \backslash D$. The aim of this note is to prove:

Theorem 1.1. In addition to the above assumptions, assume that $X$ and $Y$ are regular, and connected.

(a) If $\operatorname{Lef}(X, Y)$ holds, then (1.1) is surjective.

Received by the editors October 3, 2015 and, in revised form, February 2, 2016.

2010 Mathematics Subject Classification. Primary 14E20, 14E22.

The first author was supported by the Einstein Program.

The second author was supported by a research scholarship of the DFG ("Deutsche Forschungsgemeinschaft"). 
(b) If $\operatorname{Leff}(X, Y)$ holds, and if $Y$ intersects all effective divisors on $X$, then (1.1) is an isomorphism.

This generalizes Grothendieck's Lefschetz Theorem [7, X, Cor. 2.6, Thm. 3.10, p. 97] (see also [10. Ch. IV, Cor. 2.2, p. 177]) for $D=0$, which is then true under less restrictive assumptions.

As is well understood, if $X$ is projective and if $Y$ is a regular ample hyperplane transversal to $X \backslash D$, then $\operatorname{Lef}(X, Y)$ holds if $\operatorname{dim} X \geq 2$ and $\operatorname{Leff}(X, Y)$ holds if $\operatorname{dim} X \geq 3$ (see [7, X, Ex. 2.2, p. 92]).

We finally remark that if $k$ is algebraically closed, an alternative approach to proving Theorem 1.1 (b) would be through the theory of regular singular stratified bundles by combining [3, Thm. 5.2] with [14, Thm. 1.1].

Now assume that $X$ is possibly singular in codimension $\geq 2$ and that $D \subset X$ is a divisor such that $X \backslash D$ is smooth. Drinfeld proved in [1, Cor. C.2, Lemma C.3] that if $k$ is a finite field, and if $Y \hookrightarrow X$ is a regular projective curve intersecting the smooth locus of $D$ transversally, then the restriction functor from the category of étale covers of $X \backslash D$, tamely ramified along the smooth part of the components of $D$, to the category of étale covers of $Y \backslash(D \cap Y)$, is fully faithful. By standard arguments, we show in Proposition 6.2 that one may assume $k$ to be any field.

As one does not have at one's disposal resolution of singularities in characteristic $p>0$, it would be nice to generalize Drinfeld's theorem from dimension $Y$ equal to 1 to higher dimension, even if over an imperfect field one has to assume $X \backslash D$ to be smooth. However it is not even clear what would then be the correct formulation. In another direction, in light of Deligne's finiteness theorem [2, one would like to prove Lefschetz theorems for a fundamental group classifying coverings with bounded ramification [9, Section 3]. The abelian quotient of this theory is the content of 12 .

In Section 2 we make precise the notions of tame coverings and normal crossings divisors that we use. In Section 3 we recall Grothendieck-Murre's notion of tameness for finite maps between formal schemes and prove the first important lemma (Lemma 4.3), before we carry out the proof of Theorem 1.1] in Section 5. In Section 6] we extend Drinfeld's theorem over any field. We comment in Section 7 on the relation between Lefschetz theorems discussed in this note and Deligne's finiteness theorem.

\section{TAMELY RAMIFIED COVERINGS}

We recall the definition of a (strict) normal crossings divisor.

Definition 2.1 ([8, 1.8, p. 26]). Let $X$ be a locally noetherian scheme, and let $\left\{D_{i}\right\}_{i \in I}$ be a finite set of effective Cartier divisors on $X$. For every $x \in X$, define $I_{x}:=\left\{i \in I \mid x \in \operatorname{Supp} D_{i}\right\} \subset I$.

(a) The family of divisors $\left\{D_{i}\right\}_{i \in I}$ is said to have strict normal crossings if for every $x \in \bigcup_{i \in I} \operatorname{Supp}\left(D_{i}\right)$,

(i) the local ring $\mathcal{O}_{X, x}$ is regular,

(ii) for every $i \in I_{x}$, locally in $x$ we have $D_{i}=\sum_{j=1}^{n_{i}} \operatorname{div}\left(t_{i, j}\right)$ with $t_{i, j} \in$ $\mathcal{O}_{X, x}$, such that the set $\left\{t_{i, j} \mid i \in I_{x}, 1 \leq j \leq n_{i}\right\}$ is part of a regular system of parameters of $\mathcal{O}_{X, x}$. 
(b) The family of divisors $\left\{D_{i}\right\}_{i \in I}$ is said to have normal crossings if every $x \in \bigcup_{i \in I} \operatorname{Supp}\left(D_{i}\right)$ has an étale neighborhood $\gamma: V \rightarrow X$, such that the family $\left\{\gamma^{*} D_{i}\right\}_{i \in I}$ has strict normal crossings.

(c) An effective Cartier divisor $D$ has (strict) normal crossings if the underlying family of its irreducible components has (strict) normal crossings.

Remark 2.2. A divisor $D$ has strict normal crossings if and only if it has normal crossings and if its irreducible components are regular. One direction is 8 , Lemma 1.8.4, p. 27], while the other direction comes from (a) (ii), as the $t_{i, x} \in \mathcal{O}_{X, x}$ are local parameters.

Definition 2.3. Let $X$ be a locally noetherian, normal scheme and let $D$ be a divisor on $X$ with normal crossings. We write $\operatorname{Rev}(X)$ for the category of all finite $X$-schemes and $\operatorname{RevEt}(X)$ for the category of finite étale $X$-schemes. Following [8. 2.4.1, p. 40], we define $\operatorname{Rev}^{D}(X)$ to be the full subcategory of $\operatorname{Rev}(X)$ with objects the finite $X$-schemes tamely ramified along $D$. Recall that a finite morphism $f: Z \rightarrow X$ is called tamely ramified along $D$, if

(i) $Z$ is normal,

(ii) $f$ is étale over $X \backslash \operatorname{Supp}(D)$,

(iii) every irreducible component of $Z$ dominates an irreducible component of $X$,

(iv) for $x \in D$ of codimension 1 in $X$, and any $z \in Z$ mapping to $x$, the extension of discrete valuation rings $\mathcal{O}_{X, x} \rightarrow \mathcal{O}_{Z, z}$ is tamely ramified ([8, Def. 2.1.2, p. 30]).

The natural functors $\operatorname{RevEt}(X) \rightarrow \operatorname{Rev}^{D}(X) \rightarrow \operatorname{Rev}(X)$ are fully faithful.

Remark 2.4. The restriction functor $\operatorname{Rev}^{D}(X) \rightarrow \operatorname{RevEt}(X \backslash D)$ is fully faithful when $X$ is proper. Its essential image is the full subcategory of étale coverings of $X \backslash D$ which are tamely ramified along $D$, which, by the fundamental theorem [13. Prop. 4.2], does not depend on the choice of $X$ and is even definable on a normal compactification of $X \backslash D$. A quasi-inverse functor assigns to $Z \rightarrow X \backslash D$, étale, tame, with $Z$ connected, the normalization of $X$ in the function field of $Z$.

Remark 2.4 shows that Theorem 1.1 is equivalent to the following.

Theorem 2.5. Let $k$ be a field, let $X$ be a proper, regular, connected $k$-scheme, let $D$ be a strict normal crossings divisor on $X$, and let $Y \subset X$ be a regular, closed subscheme, such that the inverse image $\left.D\right|_{Y}$ of $D$ on $Y$ exists and is a strict normal crossings divisor.

(a) If $\operatorname{Lef}(X, Y)$ holds, then restriction induces a fully faithful functor

$$
\operatorname{Rev}^{D}(X) \rightarrow \operatorname{Rev}^{\left.D\right|_{Y}}(Y) .
$$

(b) If $\operatorname{Leff}(X, Y)$ holds and if $Y$ intersects every effective divisor on $X$, then (2.1) is an equivalence.

\section{TAMELY RAMIFIED COVERINGS OF FORMAL SCHEMES}

We recall a few definitions from [8, §3, §4].

Definition 3.1 ([8,3.1.4, 3.1.5, p. 45]). Let $\mathfrak{X}$ be a locally noetherian formal scheme. If $D$ is an effective divisor on $\mathfrak{X}$ (that is, defined by an invertible coherent sheaf of ideals in $\left.\mathcal{O}_{\mathfrak{X}},[6, \S 21]\right)$, then for any point $x \in \operatorname{Supp}(D)$, the localization $D_{x}$ 
is an effective divisor on $\operatorname{Spec} \mathcal{O}_{\mathfrak{X}, x}$. The divisor $D$ is said to have (strict) normal crossings (resp. to be regular) if $D_{x}$ is a (strict) normal crossings divisor (resp. is a regular divisor) on $\operatorname{Spec} \mathcal{O}_{\mathfrak{X}, x}$ for all $x \in \operatorname{Supp}(D)$. A finite set $\left\{D_{i}\right\}_{i \in I}$ of effective divisors on $\mathfrak{X}$ is said to have (strict) normal crossings, if for every $x \in \mathfrak{X}$ the family $\left\{\left(D_{i}\right)_{x}\right\}_{i \in I}$ has (strict) normal crossings.

Definition 3.2 ([8, 3.2.2, p. 49]). A morphism $f: \mathfrak{Y} \rightarrow \mathfrak{X}$ between two locally noetherian formal schemes is an étale covering if $f$ is finite, $f_{*} \mathcal{O}_{\mathfrak{Y}}$ is a locally free $\mathcal{O}_{\mathfrak{X}}$-module, and for all $x \in \mathfrak{X}$ the induced map of (usual) schemes $\mathfrak{Y} \times \mathfrak{X} \operatorname{Spec} k(x) \rightarrow$ $\operatorname{Spec} k(x)$ is étale. We write $\operatorname{Rev}(\mathfrak{X})$ for the category of all finite maps to $\mathfrak{X}$ and $\operatorname{RevEt}(\mathfrak{X})$ for the category of all étale coverings of $\mathfrak{X}$.

Definition 3.3 ([8, 4.1.2, p. 52]). A locally noetherian formal scheme $\mathfrak{X}$ is said to be normal if all stalks of $\mathcal{O}_{\mathfrak{X}}$ are normal. Let $\mathfrak{X}$ be normal and let $D$ be a divisor with normal crossings on $\mathfrak{X}$. A finite morphism $f: \mathfrak{Y} \rightarrow \mathfrak{X}$ is said to be a tamely ramified covering with respect to $D$, if for every $x \in \mathfrak{X}$ the finite morphism of schemes

$$
\operatorname{Spec}\left(\left(f_{*} \mathcal{O}_{\mathfrak{Y}}\right)_{x}\right) \rightarrow \operatorname{Spec}\left(\mathcal{O}_{\mathfrak{X}, x}\right)
$$

is tamely ramified along the normal crossings divisor $D_{x}$ in $\operatorname{Spec} \mathcal{O}_{\mathfrak{X}, x}$.

We write $\operatorname{Rev}^{D}(\mathfrak{X})$ for the category of tamely ramified coverings of $\mathfrak{X}$ with respect to $D$.

The first main ingredient in the proof of Theorem 2.5] is the following lifting result.

Theorem 3.4 ([8, Thm. 4.3.2, p. 58]). Let $\mathfrak{X}$ be a locally noetherian, normal formal scheme and let $\left(D_{i}\right)_{i \in I}$ be a finite set of regular divisors with normal crossings on $\mathfrak{X}$. Write $D:=\sum_{i \in I} D_{i}$. Let $\mathcal{J}$ be an ideal of definition of $\mathfrak{X}$ with the following properties:

(a) The scheme $X_{0}:=\left(\mathfrak{X}, \mathcal{O}_{\mathfrak{X}} / \mathcal{J}\right)$ is normal.

(b) The inverse images $D_{i, 0}$ on $X_{0}$ of the divisors $D_{i}$ exist, are regular, and the family $\left(D_{i, 0}\right)_{i \in I}$ has normal crossings. Write $D_{0}:=\sum_{i \in I} D_{i, 0}$.

Then the restriction functor

$$
\operatorname{Rev}(\mathfrak{X}) \rightarrow \operatorname{Rev}\left(X_{0}\right),(\mathfrak{Z} \rightarrow \mathfrak{X}) \mapsto\left(\mathfrak{Z} \times \mathfrak{X} X_{0} \rightarrow X_{0}\right)
$$

restricts to an equivalence of categories

$$
\operatorname{Rev}^{D}(\mathfrak{X}) \rightarrow \operatorname{Rev}^{D_{0}}\left(X_{0}\right) .
$$

\section{Some FACts ABOUt FORMAL COMPLETION}

The following facts are probably well known, but we could not find a reference.

Lemma 4.1. Let $A$ be an excellent ring and let $I \subset A$ be an ideal. Assume that $A^{*}:=\lim _{n} A / I^{n}$ is excellent (see Remark 4.2). Write $X:=\operatorname{Spec} A, Y:=$ $\operatorname{Spec} A / I$, and $\mathfrak{X}:=\operatorname{Spf} A^{*}$. Then $X$ is normal in some open neighborhood of $Y$ if and only if $\mathfrak{X}$ is normal.

Remark 4.2. As a special case of [6, 7.4.8, p. 203], Grothendieck asks whether $A^{*}$ is excellent whenever $A$ is. O. Gabber has proved this result unconditionally ([16, Remark 3.1.1], [11, Remark 1.2.9]). Unfortunately, to our knowledge, the proof is not yet available in written form. 
On the other hand, it is proved in [17] that if $A$ is a finitely generated algebra over a field, then $A^{*}$ is excellent. We shall apply Lemma 4.1 only in this situation.

In the sequel, the following lemma is crucially used.

Lemma $4.3([6,7.8 .3,(\mathrm{v})$, p. 215]). Let $(R, \mathfrak{m})$ be an excellent local ring and let $J \subset \mathfrak{m}$ be an ideal. Then $R$ is normal if and only if the $J$-adic completion $\lim _{i} R / J^{i}$ is normal.

We prove the main result of this section.

Proof of Lemma 4.1. We use the notation from the statement of Lemma 4.1. For a prime ideal $\mathfrak{p} \in \operatorname{Spec} A$ containing $I$, denote by $\mathfrak{p}^{*}$ the corresponding prime ideal in $A^{*}$ and also the corresponding point of $\mathfrak{X}=\operatorname{Spf}\left(A^{*}\right)$. Since the normal locus of Spec $A$ is open ([6, Scholie 7.8.3, (iv), p. 215]), we need to show that for a prime ideal $\mathfrak{p} \subset A$ containing $I$, the local ring $A_{\mathfrak{p}}$ is normal if and only if $\mathcal{O}_{\mathfrak{X}, \mathfrak{p}^{*}}$ is normal.

Let $\mathfrak{p} \in \operatorname{Spec} A$ be a prime ideal containing $I$. The canonical map of local rings $A_{\mathfrak{p}} \rightarrow A_{\mathfrak{p}^{*}}^{*}$ becomes an isomorphism $\widehat{A_{\mathfrak{p}}} \cong \widehat{A_{\mathfrak{p}^{*}}^{*}}$ after completion with respect to the maximal ideals ([15, 24.B, D, p. 173]). As both $A$ and $A^{*}$ are excellent by assumption, the same is true for the localizations $A_{\mathfrak{p}}$ and $A_{\mathfrak{p}^{*}}^{*}$. Thus, Lemma 4.3 applied to the local rings $A_{\mathfrak{p}}$ and $A_{\mathfrak{p}^{*}}^{*}$, with the topologies defined by their maximal ideals, yields that $A_{\mathfrak{p}}$ is normal if and only if $\widehat{A_{\mathfrak{p}}} \cong \widehat{A_{\mathfrak{p}^{*}}^{*}}$ is normal, if and only if $A_{\mathfrak{p}^{*}}^{*}$ is normal.

Let $A_{\mathfrak{p}^{*}}^{*} \rightarrow\left(A_{\mathfrak{p}^{*}}^{*}\right)^{*}$ be the $I$-adic completion of the localization $A_{\mathfrak{p}^{*}}^{*}$ of $A^{*}$ at $\mathfrak{p}^{*}$. It factors

$$
A_{\mathfrak{p}^{*}}^{*} \stackrel{\lambda}{\rightarrow} \mathcal{O}_{\mathfrak{X}, \mathfrak{p}^{*}} \stackrel{\mu}{\rightarrow}\left(A_{\mathfrak{p}^{*}}^{*}\right)^{*},
$$

with $\lambda$ and $\mu$ both faithfully flat $\left(\left[\underline{8}, 3.1 .2\right.\right.$, p. 44]). Indeed, for $f \in A^{*}$, write $S_{f}:=\left\{1, f, f^{2}, \ldots\right\}$ and $A_{\{f\}}$ for the $I$-adic completion of $S_{f}^{-1} A$. Then $\mathcal{O}_{\mathfrak{X}, \mathfrak{p}^{*}}=$ $\lim _{f \notin \mathfrak{p}^{*}} A_{\{f\}}([\underline{5}, 10.1 .5$, p. 182]). Faithful flatness of $\lambda$ (resp. $\mu$ ) now follows from [5. Ch. 0, 6.2.3, p. 56] together with [5, Ch. 0, 7.6.13, p. 74] (resp. [5. Ch. 0, 7.6.18, p. 75$])$.

We complete the proof: If $\mathcal{O}_{\mathfrak{X}, \mathfrak{p}^{*}}$ is normal, then by faithfully flat descent, $A_{\mathfrak{p}^{*}}^{*}$ is normal ([15, 21.E, p. 156]), and thus, as we saw above, $A_{\mathfrak{p}}$ is normal as well. Conversely, if $A_{\mathfrak{p}}$ is normal, then the excellent ring $A_{\mathfrak{p}^{*}}^{*}$ is normal, and so is its $I$-adic completion $\left(A_{\mathfrak{p}^{*}}^{*}\right)^{*}$ (Lemma 4.3). By faithfully flat descent, $\mathcal{O}_{\mathfrak{X}, \mathfrak{p}^{*}}$ is normal as well.

Corollary 4.4. Let $k$ be a field and let $X$ be a normal, separated, finite type $k$ scheme with $D \subset X$ a strict normal crossings divisor. Let $Y \subset X$ be a normal closed subscheme, such that the inverse image $\left.D\right|_{Y}$ of $D$ on $Y$ exists and is a strict normal crossings divisor, and let $X_{Y}$ be the formal completion of $X$ along $Y$. Then:

(a) The formal scheme $X_{Y}$ is normal, the inverse image $\left.D\right|_{X_{Y}}$ of $D$ on $X_{Y}$ exists and is a normal crossings divisor with regular components.

(b) The functor $\operatorname{Rev}^{\left.D\right|_{X_{Y}}}\left(X_{Y}\right) \rightarrow \operatorname{Rev}^{\left.D\right|_{Y}}(Y)$ of restriction is an equivalence.

(c) If $\mathfrak{Z} \rightarrow X_{Y}$ is a tamely ramified covering with respect to $\left.D\right|_{X_{Y}}$, then $\mathfrak{Z}$ is a normal formal scheme.

Proof. (a) $X_{Y}$ is locally noetherian and normal, according to Lemma 4.1 (here we use the fact that $X$ is of finite type over a field). By Remark 2.2, the components $\left\{D_{i}\right\}_{i \in I}$ of $D$ are regular divisors. Thus, according to [8, 4.1.4, p. 53], if $j: X_{Y} \rightarrow X$ 
is the canonical map of locally ringed spaces, then $\left(j^{*} D_{i}\right)_{i \in I}$ is a family of regular divisors with normal crossings on the formal scheme $X_{Y}$.

(b) The condition (b) of Theorem 3.4 is then fulfilled, as we assume that $\left.D\right|_{Y}$ is a strict normal crossings divisor. Thus Theorem 3.4 applies and Corollary 4.4 (b) follows.

(c) Let $f: \mathfrak{Z} \rightarrow X_{Y}$ be a tamely ramified covering. To prove that $\mathfrak{Z}$ is normal, we may assume that $X=\operatorname{Spec} A$ and $Y=\operatorname{Spec} A / I$. Let $A^{*}$ be the $I$-adic completion of $A$, so that $X_{Y}=\operatorname{Spf} A^{*}$. Let $B$ be the finite $A^{*}$-algebra such that $\mathfrak{Z}=\operatorname{Spf}(B)$. As $X_{Y}$ is normal, $A^{*}$ is also normal $([8,3.1 .3$, p. 44]). We can apply [8, Lemma 4.1 .3 , p. 52], which says that the fact that $f$ is tamely ramified with respect to $D$ is equivalent to the fact that the induced map $\operatorname{Spec} B \rightarrow \operatorname{Spec} A^{*}$ is tamely ramified with respect to the divisor on Spec $A^{*}$ corresponding to $D$. In particular, $B$ is normal. As in Lemma 4.1, for every $z \in \mathfrak{Z}$, corresponding to a prime ideal $\mathfrak{p} \subset B$ containing $I B$, we have a sequence of faithfully flat maps

$$
B_{\mathfrak{p}} \rightarrow \mathcal{O}_{\mathfrak{Z}, z} \rightarrow\left(B_{\mathfrak{p}}\right)^{*},
$$

where $(-)^{*}$ denotes $I B$-adic completion. As $A$ is of finite type over a field, $A$ is excellent, so $A^{*}$ is excellent (see Remark 4.2), and hence so are the finite $A$-algebra $B$ and its localization $B_{\mathfrak{p}}$. Lemma 4.3 implies that $\left(B_{\mathfrak{p}}\right)^{*}$ is normal, so $\mathcal{O}_{\mathfrak{Z}, z}$ is normal as well.

\section{Proof of Theorem 1.1}

We saw that Theorem 1.1 is equivalent to Theorem 2.5.

Let $X, Y, D$ be as in Theorem 2.5. Denote by $X_{Y}$ the completion of $X$ along $Y$. In Corollary 4.4 we proved that $X_{Y}$ is a normal formal scheme.

Restriction gives a sequence of functors

$$
\operatorname{Rev}(X) \rightarrow \operatorname{Rev}\left(X_{Y}\right) \rightarrow \operatorname{Rev}(Y) .
$$

According to [8, Cor. 4.1.4, p. 53] and Corollary 4.4, this sequence restricts to

$$
\operatorname{Rev}^{D}(X) \stackrel{F_{1}}{\longrightarrow} \operatorname{Rev}^{\left.D\right|_{X}}\left(X_{Y}\right) \stackrel{F_{2}}{\longrightarrow} \operatorname{Rev}^{\left.D\right|_{Y}}(Y) .
$$

We already saw in Corollary 4.4 that $F_{2}$ is an equivalence. It remains to show that $F_{1}$ is fully faithful if $\operatorname{Lef}(X, Y)$ holds, and that $F_{1}$ is an equivalence if $\operatorname{Leff}(X, Y)$ holds and $Y$ meets every effective divisor on $X$.

The fact that enables us to use $\operatorname{Lef}(X, Y)$ and $\operatorname{Leff}(X, Y)$, which are conditions involving coherent locally free sheaves, is that tame coverings are flat. More precisely, an object $Z \rightarrow X$ of $\operatorname{Rev}^{D}(X)$ is a flat morphism according to [8, Cor. 2.3.5, p. 39], and an object $\mathfrak{Z} \rightarrow X_{Y}$ of $\operatorname{Rev}^{\left.D\right|_{Y}}\left(X_{Y}\right)$ is a flat morphism of formal schemes ([8, 3.1.7, p. 47] together with [8, 4.1.3, p. 52]).

If $f: Z \rightarrow X$ is a tamely ramified cover with respect to $D$, then $f$ is flat, so $f_{*} \mathcal{O}_{Z}$ is a locally free $\mathcal{O}_{X}$-module of finite rank. Morphisms in $\operatorname{Rev}^{D}(X)$ are thus defined by morphisms of $\mathcal{O}_{X}$-algebras which are locally free $\mathcal{O}_{X}$-modules. Assuming $\operatorname{Lef}(X, Y)$, this means that for every pair of objects $Z, Z^{\prime} \rightarrow X$ of $\operatorname{Rev}^{D}(X)$, the restriction map

$$
\operatorname{Hom}_{X}\left(Z, Z^{\prime}\right) \stackrel{\cong}{\rightarrow} \operatorname{Hom}_{X_{Y}}\left(Z_{Y}, Z_{Y}^{\prime}\right)
$$

is bijective. This shows that $F_{1}$ is fully faithful. 
An object $f: \mathfrak{Z} \rightarrow X_{Y}$ of $\operatorname{Rev}^{\left.D\right|_{X_{Y}}}\left(X_{Y}\right)$ is determined by the locally free $\mathcal{O}_{X_{Y}}$ algebra $f_{*} \mathcal{O}_{\mathfrak{Z}}$. Assuming $\operatorname{Leff}(X, Y)$, for every such object there exists an open subset $U \subset X$ containing $Y$ and a locally free sheaf $\mathcal{A}$ on $U$ such that $\left.\mathcal{A}\right|_{X_{Y}} \cong f_{*} \mathcal{O}_{\mathfrak{Z}}$. As $\operatorname{Lef}(X, Y)$ holds, we can lift the algebra structure from $f_{*} \mathcal{O}_{\mathfrak{Z}}$ to $\mathcal{A}$. Indeed, the global section of $\left(f_{*} \mathcal{O}_{\mathfrak{Z}} \otimes_{\mathcal{O}_{X_{Y}}} f_{*} \mathcal{O}_{\mathfrak{Z}}\right)^{\vee} \otimes_{\mathcal{O}_{X_{Y}}} f_{*} \mathcal{O}_{\mathfrak{Z}}$ defining the algebra structure lifts to a global section of $\left(\mathcal{A} \otimes_{\mathcal{O}_{U}} \mathcal{A}\right)^{\vee} \otimes_{\mathcal{O}_{U}} \mathcal{A}$, endowing $\mathcal{A}$ with an $\mathcal{O}_{U}$-algebra structure. Write $Z:=\operatorname{Spec} \mathcal{A}$. We obtain a finite, flat morphism $g: Z \rightarrow U$ which restricts to $f: \mathfrak{Z} \rightarrow X_{Y}$.

According to Corollary 4.4 (c) the formal scheme $\mathfrak{Z}$ is normal. We can identify $\mathfrak{Z}$ with the formal completion of $Z$ along the closed subset $g^{-1}(Y)$ ([5, Cor. 10.9.9, p. 200]). As $Z$ is excellent, Lemma 4.1 implies that $Z$ is normal in an open neighborhood of $g^{-1}(Y)$. Now the assumptions of [8, Cor. 4.1.5, p. 54] are satisfied, from which it follows that there is an open subset $V \subset U \subset X$ containing $Y$, such that $g_{V}: Z \times_{U} V \rightarrow V$ is tamely ramified with respect to $V \cap D$. Lemma 5.1 shows that $g$ extends to an object of $\operatorname{Rev}^{D}(X)$ lifting $f$.

Lemma 5.1. Assume that $Y$ meets every effective divisor on $X$. If $U \subset X$ is an open subset containing $Y$, then restriction induces an equivalence

$$
\operatorname{Rev}^{D}(X) \stackrel{\cong}{\rightarrow} \operatorname{Rev}^{D \cap U}(U) \text {. }
$$

Proof. By assumption, $Y$ intersects every effective divisor on $X$, so

$$
\operatorname{codim}_{X}(X \backslash U)>1 \text {. }
$$

Given a finite morphism $Z \rightarrow U$, tamely ramified over $U \cap D$, the normalization $Z_{X} \rightarrow X$ of $X$ in $Z$ is finite étale over $X \backslash D$, as $X$ is regular ("purity of the branch locus") and tamely ramified over $D$. This yields a quasi-inverse functor to the restriction functor (5.1).

Remark 5.2. If $k$ has characteristic 0 , then the quotient homomorphism $\pi_{1}(X \backslash D) \rightarrow \pi_{1}^{\text {tame }}(X \backslash D)$ is an isomorphism. For the theorem corresponding to Theorem 1.1 for the topological fundamental group when $k=\mathbb{C}$, assuming $X$ is smooth but not necessarily a normal crossings compactification of $X \backslash D$, we refer to [4, 1.2, Remarks, p. 153]. Of course, by the comparison isomorphisms, the topological theorem implies Theorem 1.1 (a).

\section{DRINFELD'S THEOREM}

Theorem 6.1 (Drinfeld's theorem, 1, Prop. C.2]). Let $X$ be a geometrically irreducible projective variety over a finite field $k$, let $D \subset X$ be a divisor, and let $\Sigma \subset D$ be a closed subscheme of codimension $\geq 1$ in $D$, such that $X \backslash \Sigma$ and $D \backslash \Sigma$ are smooth. Then any smooth, geometrically irreducible curve $Y \subset X$ which intersects $D$ in $D \backslash \Sigma$, and is transversal to $D \backslash \Sigma$, has the property that the restriction to $Y \backslash D \cap Y$ of any finite étale connected cover of $X \backslash D$, which is tamely ramified along $D \backslash \Sigma$, is connected.

That such curves exist can be deduced from Poonen's Bertini theorem over finite fields. They are constructed as global complete intersections of high degree (see [1, C.2]). We remark:

Proposition 6.2. Theorem 6.1 remains true over any field $k$, and there exists $Y \subset X$ satisfying the conditions of Theorem 6.1 . 
Proof. The data $(X, D, \Sigma)$ are defined over a ring of finite type $R$ over $\mathbb{Z}$, say $\left(X_{R}, D_{R}, \Sigma_{R}\right)$, such that for any closed point $s \in \operatorname{Spec}(R)$, the restriction $\left(X_{s}, D_{s}, \Sigma_{s}\right)$ fulfills the assumptions of Theorem 6.1. Fix such an $s$, and a $Y_{s}$ as in the theorem. The equations of $Y_{s}$ lift to an open subset of $\operatorname{Spec}(R)$ containing $s$. Shrinking $\operatorname{Spec}(R)$, the lift $Y_{R}$ intersects $D_{R}$ in $D_{R} \backslash \Sigma_{R}$ and is transversal to $D_{R} \backslash \Sigma_{R}$, thus $Y:=Y_{R} \otimes_{R} k$ intersects $D$ in $D \backslash \Sigma$ and is transversal to $D \backslash \Sigma$, and for all closed points $t \in \operatorname{Spec}(R), Y_{t}$ intersects $D_{t}$ in $D_{t} \backslash \Sigma_{t}$ and is transversal to $D_{t} \backslash \Sigma_{t}$.

Now let $h: V \rightarrow X \backslash D$ be a connected finite étale cover, tamely ramified along $D \backslash \Sigma$. Writing $W:=V \times_{X \backslash D}(Y \backslash D)$, our goal is to prove that $W$ is connected. Let $h^{\prime}: V^{\prime} \rightarrow X$ be the normalization of $X$ in $V$, and let $g^{\prime}: W^{\prime} \rightarrow Y$ be the normalization of $Y$ in $W$. By assumption, $D \cap Y$ is finite étale over $k$, so we can write $D \cap Y=\coprod_{i=1}^{n} \operatorname{Spec}\left(k\left(x_{i}\right)\right)$ with $k \subset k\left(x_{i}\right)$ finite separable. As $h^{\prime}$ is tamely ramified with respect to $D \backslash \Sigma$, according to Abhyankar's Lemma (88, Cor. 2.3.4, p. 39]), there are affine étale neighborhoods $\eta_{i}: U_{i} \rightarrow X \backslash \Sigma$ of $x_{i}, i=1, \ldots, n$, such that for every $i, \eta_{i} \times h^{\prime}: U_{i} \times_{X \backslash \Sigma} V^{\prime} \rightarrow U_{i}$ is isomorphic to a disjoint union of Kummer coverings; we have a diagram

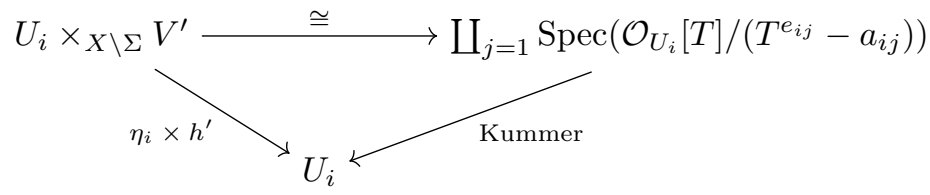

where the $e_{i j}$ are prime to $\operatorname{char}(k)$, and the $a_{i j} \in H^{0}\left(U_{i}, \mathcal{O}_{U_{i}}\right)$ are regular and units outside of $(D \backslash \Sigma) \times(X \backslash \Sigma) U_{i}$.

Shrinking $\operatorname{Spec}(R)$, the data $\left(X, \Sigma, Y, D, h, h^{\prime}, g^{\prime}, \eta_{i}, a_{i j}\right)$ and the isomorphisms from (6.1) are defined over $R$; denote by $\left(X_{R}, \Sigma_{R}, \ldots\right)$ the corresponding models over $R$. Shrinking Spec $R$ again, we may assume that $h_{R}^{\prime}: V_{R}^{\prime} \rightarrow X_{R} \backslash \Sigma_{R}$ is étale over $X_{R} \backslash D_{R}$, that $g_{R}^{\prime}: W_{R}^{\prime} \rightarrow Y_{R}$ is étale over $Y_{R} \backslash D_{R}$, and that $Y_{s}$ is smooth and geometrically irreducible for all closed points $s \in \operatorname{Spec}(R)$.

Moreover, as $D \backslash \Sigma$ is smooth and as $Y$ intersects $D$ transversally and in $D \backslash \Sigma$, we may assume that $D_{R} \cap Y_{R}$ is finite étale over $\operatorname{Spec} R$, and that

$$
\left.\coprod_{i} \eta_{i, R}\right|_{Y_{R} \cap D_{R}}: \coprod_{i} U_{i, R} \times_{X_{R}}\left(Y_{R} \cap D_{R}\right) \rightarrow\left(Y_{R} \cap D_{R}\right)
$$

is surjective.

For $s \in \operatorname{Spec} R$ a closed point of residue characteristic prime to the exponents $e_{i j}$ from (6.1), the morphisms $\left.\eta_{i, s}\right|_{Y_{s}}: U_{i, s} \times_{X_{s}} Y_{s} \rightarrow Y_{s} \backslash \Sigma_{s}$ are étale neighborhoods of the points of $Y_{s}$ lying on $D_{s} \backslash \Sigma_{s}$, and each $g_{s}^{\prime} \times \eta_{i, s}$ is isomorphic to a disjoint union of Kummer coverings. Thus, again by Abhyankar's Lemma (8), Cor. 2.3.4, p. 39]), $g_{s}^{\prime}: W_{s}^{\prime} \rightarrow Y_{s} \backslash \Sigma_{s}$ is tamely ramified along $\left(Y_{s} \cap D_{s}\right) \backslash \Sigma_{s}$.

The morphism $\lambda: W_{R}^{\prime} \rightarrow \operatorname{Spec}(R)$ is projective; thus shrinking $\operatorname{Spec}(R)$ again, one has base change for $\lambda_{*} \mathcal{O}_{W_{R}^{\prime}}$. By Theorem 6.1, $H^{0}\left(W_{s}^{\prime}, \mathcal{O}_{W_{s}^{\prime}}\right)=k(s)$. Thus $\lambda_{*} \mathcal{O}_{W_{R}^{\prime}}$ is an $R$-projective module of rank one; thus by base change again, $H^{0}\left(W^{\prime}, \mathcal{O}_{W^{\prime}}\right)=k$, and $W$ is connected. This finishes the proof.

Remark 6.3. Recall that in [13, tame coverings of $X \backslash D$ in Theorem 6.1 are defined, and more generally, tame coverings of regular schemes of finite type over an excellent, integral, pure-dimensional scheme. They build a Galois category, with 
Galois group $\pi_{1}^{\text {tame }}(X \backslash D, \bar{y})$, which is a full subcategory of the Galois category of the covers considered by Drinfeld in Theorem 6.1, where he considered the tameness condition only along $D \backslash \Sigma$. Thus for $Y$ as in Proposition 6.2, the functoriality homomorphism $\pi_{1}^{\text {tame }}(Y \backslash D \cap Y, \bar{y}) \rightarrow \pi_{1}^{\text {tame }}(X \backslash D, \bar{y})$ is surjective. As in Remark 5.2, we observe that this latter formulation in characteristic 0 follows from [4, 1.2, Remarks, p. 153].

\section{Comments}

Drinfeld's theorem holds even if $X \backslash D$ does not have a good compactification. This is in contrast to Theorem 1.1. It would be nice to have a version of Theorem 1.1 (b) which does not require the existence of a good compactification.

Let $X$ be a smooth projective, connected $k$-scheme, and let $D$ be a strict normal crossings divisor. If $k$ is perfect, in [12, a quotient $\pi_{1}^{\mathrm{ab}}(X, D)$ of $\pi_{1}^{\mathrm{e} t \text {,ab }}(X \backslash D)$ is defined. There are canonical quotient homomorphisms

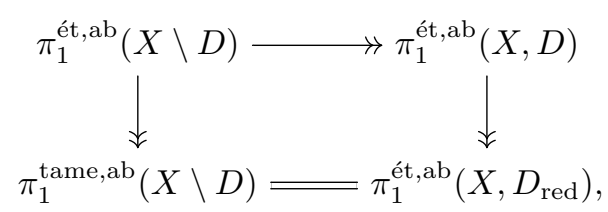

where the groups in the left column are the abelianizations of the étale and tame fundamental group. Let $\ell$ be a prime number different from $\operatorname{char}(k)$. The $\overline{\mathbb{Q}}_{\ell^{-}}$ lisse sheaves of rank one, which have ramification bounded by $D$ in the sense of [2, Def. 3.6], are precisely the irreducible $\ell$-adic representations of $\pi_{1}^{\text {ét,ab }}(X, D)$. The main result of 12 is a Lefschetz theorem in the form of Theorem [1.1] for $\pi_{1}^{\text {ét, ab }}(X, D)$.

One would wish to have a general notion of fundamental group $\pi_{1}^{\text {ét }}(X, D)$ encoding finite étale covers with ramification bounded by $D$, and to show a Lefschetz theorem similar to Theorem 6.1 for them. This would shed new light on Deligne's finiteness theorem [2] over a finite field.

\section{ACKNOWLEDGEMENTS}

The authors thank Moritz Kerz for discussions on the topic of this note at the time [2] was written. They also posed the problem solved in this note to Sina Rezazadeh, who unfortunately has left mathematics. The second author wishes to thank Harvard University for its hospitality during his visit.

\section{REFERENCES}

[1] Vladimir Drinfeld, On a conjecture of Deligne (English, with English and Russian summaries), Mosc. Math. J. 12 (2012), no. 3, 515-542, 668. MR3024821

[2] Hélène Esnault and Moritz Kerz, A finiteness theorem for Galois representations of function fields over finite fields (after Deligne), Acta Math. Vietnam. 37 (2012), no. 4, 531-562. MR.3058662

[3] David Gieseker, Flat vector bundles and the fundamental group in non-zero characteristics, Ann. Scuola Norm. Sup. Pisa Cl. Sci. (4) 2 (1975), no. 1, 1-31. MR0382271

[4] Mark Goresky and Robert MacPherson, Stratified Morse theory, Ergebnisse der Mathematik und ihrer Grenzgebiete (3) [Results in Mathematics and Related Areas (3)], vol. 14, SpringerVerlag, Berlin, 1988. MR932724 
[5] Alexander Grothendieck, Éléments de géométrie algébrique (rédigès avec la collaboration de Jean Dieudonné): I. Le langage des schémas, Inst. Hautes Études Sci. Publ. Math. (1960), no. 4, 228. MR0217083 (36 \#177a)

[6] Éléments de géométrie algébrique. IV: Étude locale des schémas et des morphismes de schémas., Publ. Math. IHES 32 (1967).

[7] Alexander Grothendieck, Cohomologie locale des faisceaux cohérents et théorèmes de Lefschetz locaux et globaux (SGA 2) (French), Documents Mathématiques (Paris) [Mathematical Documents (Paris)], 4, Société Mathématique de France, Paris, 2005. Séminaire de Géométrie Algébrique du Bois Marie, 1962; Augmenté d'un exposé de Michèle Raynaud [with an exposé by Michèle Raynaud]; with a preface and edited by Yves Laszlo; revised reprint of the 1968 French original. MR2171939

[8] Alexander Grothendieck and Jacob P. Murre, The tame fundamental group of a formal neighbourhood of a divisor with normal crossings on a scheme, Lecture Notes in Mathematics, Vol. 208, Springer-Verlag, Berlin-New York, 1971. MR.0316453

[9] Shinya Harada and Toshiro Hiranouchi, Smallness of fundamental groups for arithmetic schemes, J. Number Theory 129 (2009), no. 11, 2702-2712, DOI 10.1016/j.jnt.2009.03.010. MR2549526

[10] Robin Hartshorne, Ample subvarieties of algebraic varieties, Lecture Notes in Mathematics, Vol. 156, Springer-Verlag, Berlin-New York, 1970. Notes written in collaboration with C. Musili. MR0282977

[11] Kiran S. Kedlaya, Good formal structures for flat meromorphic connections, II: excellent schemes, J. Amer. Math. Soc. 24 (2011), no. 1, 183-229, DOI 10.1090/S0894-0347-201000681-9. MR2726603

[12] Moritz Kerz and Shuji Saito, Lefschetz theorem for abelian fundamental group with modulus, Algebra Number Theory 8 (2014), no. 3, 689-701, DOI 10.2140/ant.2014.8.689. MR3218806

[13] Moritz Kerz and Alexander Schmidt, On different notions of tameness in arithmetic geometry, Math. Ann. 346 (2010), no. 3, 641-668, DOI 10.1007/s00208-009-0409-6. MR2578565

[14] Lars Kindler, Regular singular stratified bundles and tame ramification, Trans. Amer. Math. Soc. 367 (2015), no. 9, 6461-6485, DOI 10.1090/S0002-9947-2014-06143-6. MR.3356944

[15] Hideyuki Matsumura, Commutative algebra, 2nd ed., Mathematics Lecture Note Series, vol. 56, Benjamin/Cummings Publishing Co., Inc., Reading, Mass., 1980. MR575344

[16] Michael Temkin, Desingularization of quasi-excellent schemes in characteristic zero, Adv. Math. 219 (2008), no. 2, 488-522, DOI 10.1016/j.aim.2008.05.006. MR2435647

[17] Paolo Valabrega, On the excellent property for power series rings over polynomial rings, J. Math. Kyoto Univ. 15 (1975), no. 2, 387-395. MR.0376677

FB Mathematik und Informatik, Freie Universität Berlin, Arnimallee 3, 14195 Berlin, Germany

E-mail address: esnault@math.fu-berlin.de

Department of Mathematics, Harvard University, Science Center, One Oxford Street, Cambridge, Massachusetts 02138

E-mail address: kindler@math.harvard.edu 